\title{
Study of the resistance, porosity, and migration of chloride ions of BAP- based crushing sand
}

\section{Etude de la résistance, la porosité, et migration des ions chlorures des BAP à base de sable de concassage}

\author{
Benyamina Smain ${ }^{1,2,3}$, Siham Kamali-Bernard ${ }^{2}$, Kenai Said $^{3}$, Menadi Belkacem ${ }^{3}$ \\ ${ }^{1}$ Département de Science de la matière, Université de Djillali Bounaama, Kemis Miliana, Algérie \\ ${ }^{2}$ Laboratoire de Génie civil et Génie Mécanique, Institut des sciences Appliquées, Rennes, 35708, France \\ ${ }^{3}$ Laboratoire de Matériaux et de génie civil, Département de génie civil, Université de Blida 1, Algérie
}

\begin{abstract}
Self-compacting concretes (SCC), are hyper-fluid concretes, placed without vibration and are considered as one of the most important innovations of the last decade in construction. SCCs offer many advantages, due to their exceptional characteristics of flow and filling of formwork. Their compositions require a large quantity of fines in order to limit bleeding and segregation. Hence, the use of crushed sand (SC), rich in limestone fines (CF) in the manufacture of self-placing concretes (SCC), can be considered as an alternative source of fillers. These sands reduce the cost of SCC by reducing the high demand for fillers on the one hand and on the other hand, obtaining SCC with good physical and mechanical properties. The main purpose of this paper is to examine the effect of different percentages $(0,5,10,15$, and $20 \%)$ of (CF) in crushed sand on SCC performance. The evolution of the compressive strength, the porosity accessible to water and the migration coefficient of the chloride ions were evaluated. The Okamura method was used for the formulation of all SCC mixtures. Sand/mortar (S/M), water/cement (W/C) ratios and superplasticizer content were kept constant. The results show that (CF) reduce the compressive strength but contribute to the reduction of porosity and migration of chloride ions.
\end{abstract}

Key word: SCC, crushed sand, limestone, compressive strengths, porosity, migration of chloride ions.

\begin{abstract}
Résumé. Les bétons autoplaçants (BAP), sont des bétons hyper fluides, mis en œuvre sans vibration, constituant l'une des plus importantes innovations de la dernière décennie en matière de construction. Ces bétons offrent de multiples avantages, liés à leurs caractéristiques exceptionnelles d'écoulement et de remplissage des coffrages. Leurs compositions nécessitent une quantité importante de fines afin de limiter le ressuage et la ségrégation. L'utilisation des sables de concassage (SC), riche en fines calcaires (FC) dans la fabrication des bétons autoplaçants (BAP), peut être considérer comme une source alternative de fillers. Ces sables diminuent le coût des BAP par réduction de la demande élevée en fillers d'une part, et d'autre part l'obtention des BAP avec des bonnes propriétés physiques et mécaniques. Le but principal de cette étude, est d'examiner l'effet de différents pourcentages $(0,5,10,15$, et $20 \%)$ de (FC) dans le sable de concassage sur les performances des BAP. L'évolution de la résistance à la compression, la porosité accessible à l'eau et le coefficient de migration des ions de chlorures ont été évalués. La méthode d'Okamura a été utilisée pour la formulation de toutes les mélanges des BAP. Les rapports sable / mortier $(\mathrm{S} / \mathrm{M})$, eau / ciment $(\mathrm{E} / \mathrm{C})$ et la teneur en superplastifiant ont été maintenus constants. Les résultats montrent, que les fines accélèrent les résistances à la compression aux premiers âges, et contribuent à la diminution de la porosité et la migration des ions de chlorures.
\end{abstract}

Mots clés : BAP, sable de concassage ; fines calcaires, résistance, porosité, migration des chlorures. 


\section{Introduction}

Les bétons autoplaçants (BAP), sont des bétons extrêmement fluides stables et homogènes, et qu'ils se mettent en place sous l'effet de leur poids propre sans aucun apport d'énergie. Ils peuvent être coulés dans des espaces trop ferraillés et de formes très complexes. Leurs compositions nécessitent certaines spécificités dont un volume de pâte élevé ce qui augmente leur coût. Donc l'utilisation des fines issues de concassage des sables présente un enjeu important pour des raisons à la fois économiques et environnementales.

Les travaux de recherches menés sur des mortiers ou bétons montrent l'effet bénéfique des fines sur leurs comportements [1-4]. Cependant ces études montrent que les interactions multiples et imprévisibles qui peuvent exister entre les fillers et les autres constituants peuvent entraîner des comportements inattendus.

Cette étude contribue à l'examen des performances des BAP, formulés à partir des sables de concassage contenant différentes teneurs en fines. On se basant sur la méthode d'Okamura [5], cinq mélanges de BAP ont été confectionnés dont les rapports $\mathrm{E} / \mathrm{C}=0.4, \mathrm{~S} / \mathrm{M}=0.5$, $\mathrm{Sp}=0.8$, soient fixe. Le sable $(\mathrm{SC})$ a été tamisé $(80 \mu \mathrm{m})$ puis substitué par $(0,5,10,15$ et $20 \%)$ de fines calcaire en masse. L'effet de la teneur en fines dans le sable (SC) sur le développement de la résistance à la compression, la porosité accessible à l'eau, la migration des ions de chlorures pour chaque mélange de BAP est évalué.

\section{Matériaux et essais}

\subsection{Matériaux utilisés}

Deux sables ont été utilisés, un sable grossier concassé calcaire (SC), et un sable fin siliceux (SS), d'une densité de $2.63,2.54$ et un coefficient d'absorption d'eau de 0.38 et $0.2 \%$, respectivement. Les fines calcaires (FC) sont issues après passage du sable de concassage (SC) dans un tamis de $80 \mu \mathrm{m}$. Les courbes granulométriques des deux sables utilisés sont présentées sur la figure 1

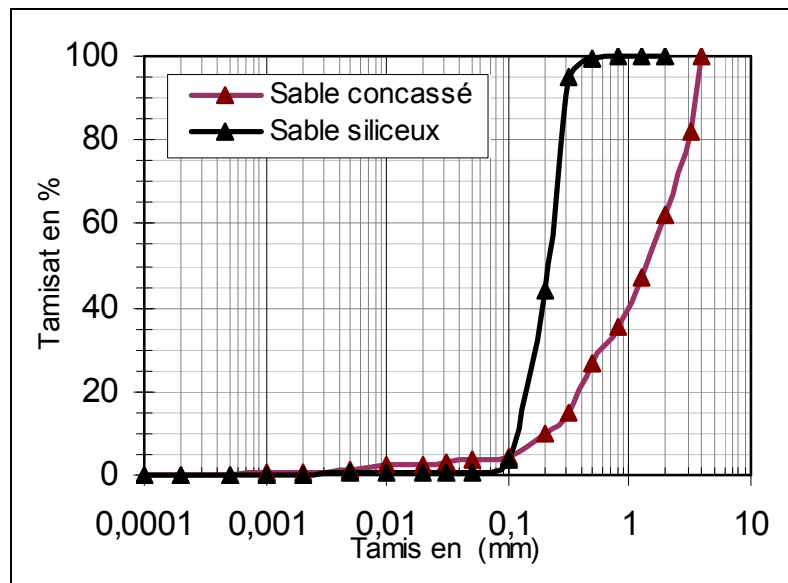

Fig. 1. Courbe granulométriques des SC et SS
L'analyse chimique du sable concassé et les fines calcaires est donné dans le tableau 1. Deux types de graviers ont été employés (G3/8 et G8/15), d'une densité apparente de 2.62 et 2.63 et d'un coefficient d'absorption de 0,20 et $0,30 \%$, respectivement.

Pour la formulation des différents mélanges des BAP, un seul ciment CEM I 42,5, d'une finesse de $3000 \mathrm{~cm}^{2} / \mathrm{g}$ et une densité de 3,15 est_utilisé.

Tableau 1. Propriétés chimiques des SC et leur FC

\begin{tabular}{|c|c|c|}
\hline $\begin{array}{c}\text { (\%) des } \\
\text { Oxydes }\end{array}$ & $\begin{array}{c}\text { Sable } \\
\text { concassé } \\
\text { (SC) }\end{array}$ & $\begin{array}{c}\text { Fines } \\
\text { calcaires } \\
\text { (FC) }\end{array}$ \\
\hline $\mathrm{SiO} 2$ & 4.51 & 1.78 \\
\hline $\mathrm{A} 2 \mathrm{O} 3$ & 1.09 & 0.79 \\
\hline $\mathrm{Fe} 2 \mathrm{O} 3$ & $\mathrm{O} .54$ & 0.34 \\
\hline $\mathrm{CaO}$ & 50.68 & 54.3 \\
\hline $\mathrm{MgO}$ & 1.01 & 0.20 \\
\hline $\mathrm{K} 2 \mathrm{O}$ & ----- & ---- \\
\hline $\mathrm{Na} 2 \mathrm{O}$ & ----- & ----- \\
\hline $\mathrm{H} 2 \mathrm{O}$ & ----- & 2.8 \\
\hline $\mathrm{SO} 3$ & traces & ----- \\
\hline Perte au feu & 41.04 & 42.5 \\
\hline
\end{tabular}

Un superplastifiant (MED Flow 145) à base de polycarboxylate avec une densité de $1.05 \pm 0.02 \mathrm{~g} / \mathrm{cm}^{3}$ est également utilisé pour toute la formulation des BAP.

\subsection{Essais réalisés}

La formulation des BAP est réalisée selon la méthode d'Okamura [5]. En total cinq mélanges ont été préparés. Les quantités et dosage pour chaque mélange sont donnés dans le tableau 2.

Tableau 2. Dosage et poids des constituants des BAP

\begin{tabular}{|c|l|l|l|l|l|}
\hline \multicolumn{6}{|c|}{$\mathrm{E} / \mathrm{C}=0.4, \mathrm{~S} / \mathrm{M}=0.5 \mathrm{G} / \mathrm{S}=0,8, \mathrm{Sp}=0,8$} \\
\hline Poids $(\mathrm{kg})$ & BAP0 & BAP5 & BAP10 & BAP15 & BAP20 \\
\hline Gravier 8/15 & 295 & 295 & 295 & 295 & 295 \\
\hline Gravier 3/8 & 443 & 443 & 443 & 443 & 443 \\
\hline Sable (SC) & 600 & 570 & 540 & 510 & 480 \\
\hline Sable (SS) & 323 & 323 & 323 & 323 & 323 \\
\hline FC & 0 & 30 & 60 & 90 & 120 \\
\hline Ciment & 490 & 460 & 430 & 400 & 370 \\
\hline E & 196 & 196 & 196 & 196 & 196 \\
\hline SP & 6,86 & 6,86 & 6,86 & 6,86 & 6,86 \\
\hline
\end{tabular}

Les essais de résistance en compression ont été réalisés sur des éprouvettes cubiques $100 \times 100 \times 100 \mathrm{~mm}^{3}$. Les éprouvettes testées ont été conservées dans l'eau dès leur démoulage jusqu'à l'échéance concerné (Fig.2). 


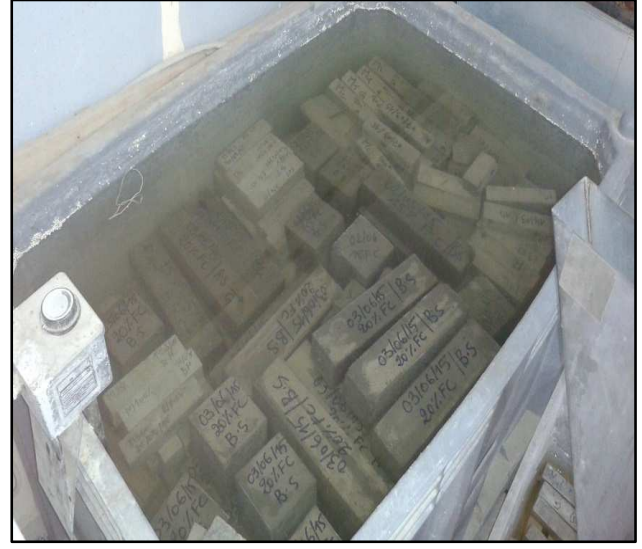

Fig.2. Conservation des éprouvettes

Les mesures de porosité accessible à l'eau ont été effectués conformément à la norme NF EN 18-459 [6] qui préconise d'abord une saturation sous vide des échantillons suivi par pesée hydrostatique puis un séchage à $105^{\circ} \mathrm{C}$. Chaque essai a été réalisé sur deux prélèvements de $50 \mathrm{~mm}$ d'épaisseur découpé au centre d'éprouvettes cylindriques 100x200 mm.

Des essais accélérés de migration des ions chlorures par champ électrique ont été réalisés. La tension appliquée aux bornes des échantillons est de $30 \mathrm{~V}$. Les cellules d'essai utilisées sont illustrées dans la figure 3 . Le coefficient de diffusion effectif De $\left(\mathrm{m}^{2} / \mathrm{s}\right)$ est déterminé selon la norme XP P 18-462 [7].

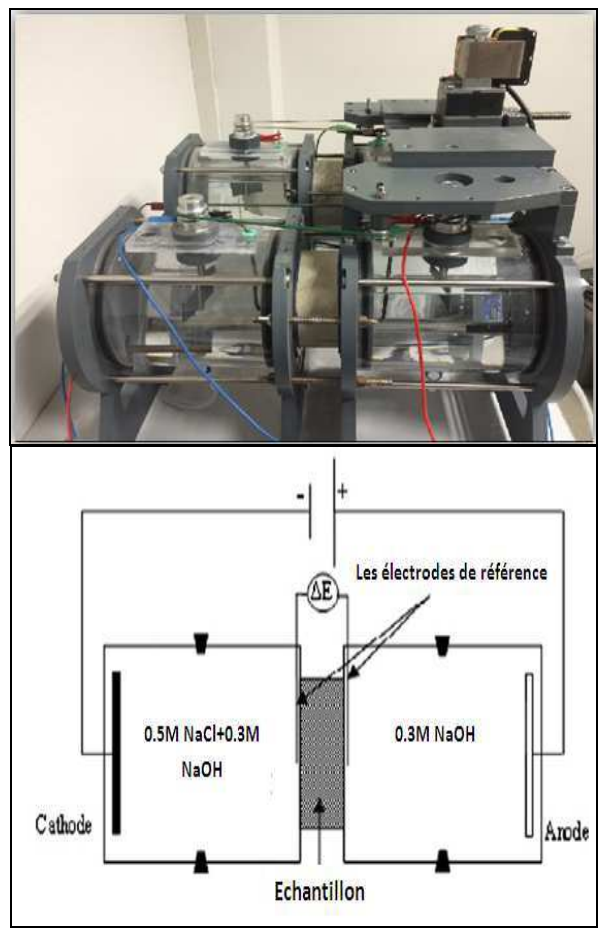

Fig. 3. Essai de migration des ions de chlorures

\section{Résultats}

\subsection{Résistance à la compression}

L'évolution de la résistance en compression à l'âge de 28 jours en fonction de la teneur des fines calcaires est donnée en figure 4. Les résultats montrent que pour tous les BAP étudiés, la résistance à la compression diminue avec l'augmentation du taux de fines dans le sable de concassage. Une résistance à la compression de $71,8 \mathrm{MPa}$ est obtenue pour un BAP de $0 \%$ de fines calcaires, puis une chute linéaire jusqu'au $65 \mathrm{MPa}$ pour un BAP de $20 \%$ de fines. Cette réduction peut être attribuée à l'effet de dilution des fines calcaires $[1,8]$.

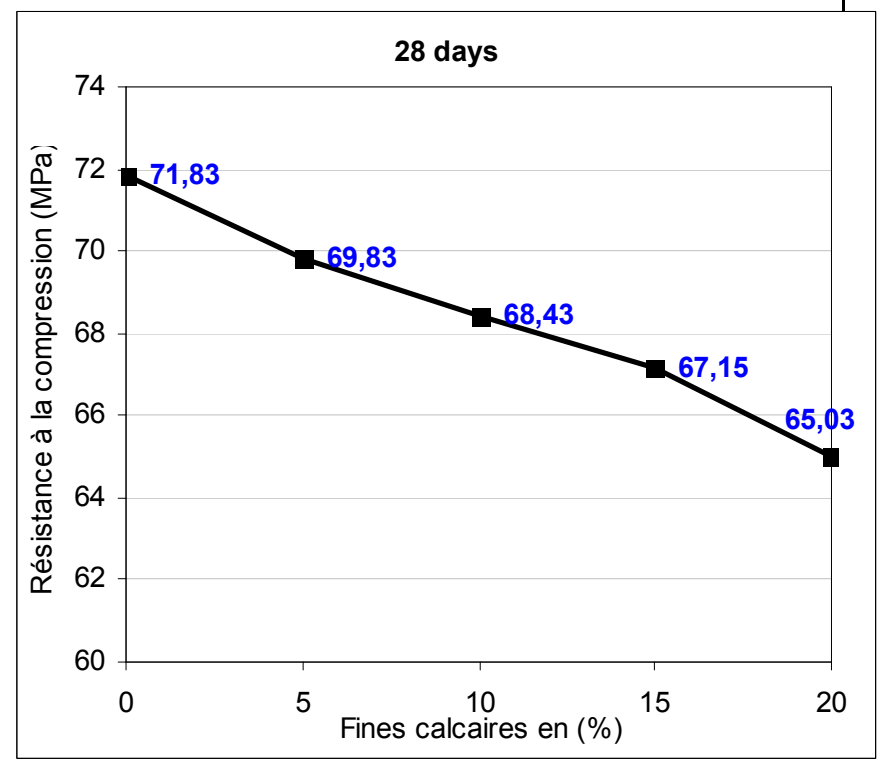

Fig. 4. Evolution de la résistance à la compression en fonction du taux de fines

\subsection{La porosité accessible à l'eau}

D'après la figure 5, les valeurs maximales des absorptions d'eau sont constatées pour le béton autoplaçant à base de sable de concassage de $0 \%$ de fines calcaires, avec une porosité de $15.22 \%$.

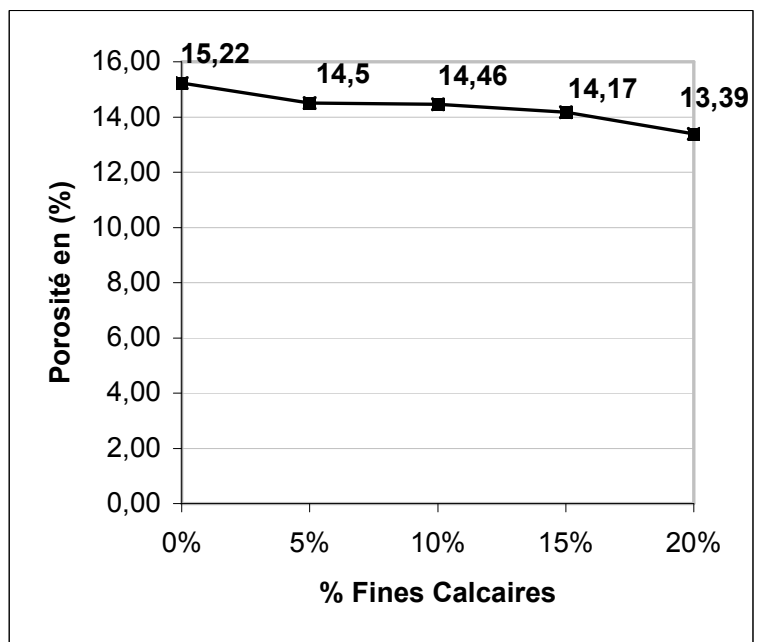

Fig. 5. Essai de migration des ions de chlorures 
Cette porosité diminue avec l'augmentation des taux de fines. A titre d'exemple, avec un BAP de $20 \%$ de fines, on remarque une réduction de la porosité de $12 \%$. Ce comportement est expliqué par l'influence des fines sur la compacité des BAP et par conséquent sur la porosité.

\subsection{Migration des ions chlorures}

L'évolution de la concentration en chlorures dans les différents mélanges des BAP en fonction du pourcentage des fines dans le sable concassé est illustrée dans la figure 6 .

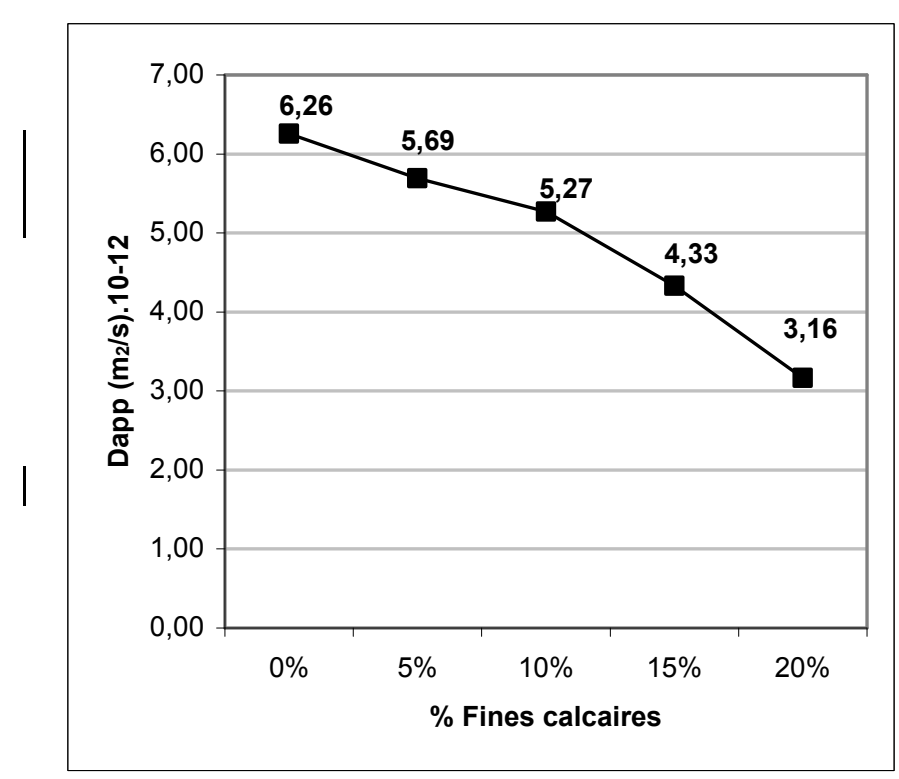

Fig. 6. Essai de migration des ions de chlorures

Les résultats montrent l'effet positif des fines calcaires sur la diminution de migration des ions chlorures dans les BAP. Le coefficient de diffusion obtenu pour le BAP de $0 \%$ de fines est égal à égale à $6.26 \times 10^{-12} \mathrm{~m}^{2} / \mathrm{s}$ alors qu'il est égal à $3.16 \times 10^{-12} \mathrm{~m}^{2} / \mathrm{s}$ pour le BAP de $20 \%$ de fines. Cette diminution est presque linéaire pour tous les mélanges de BAP. Selon cette étude il est remarqué qu'une augmentation du taux de fines de 0 à $20 \%$ en masse dans le sable concassé provoque une diminution du coefficient de migration des chlorures de $3.12 \times 10^{-12} \mathrm{~m}^{2} / \mathrm{s}$.

\section{Conclusion}

Selon cette étude qui est portée sur l'influence des sables de concassage notamment les fines calcaires sur les performance des BAP en terme de résistances mécaniques et indicateurs de durabilité à savoir porosité accessible à l'eau et migration des ions de chlorures on peut conclure ce qui suit :

- La substitution d'une partie de sable de concassage par leurs filler calcaires diminue la résistance à la compression. Une réduction de 6.8 $\mathrm{MPa}$ est notée pour un BAP avec un remplacement de $20 \%$ de fines calcaires en comparaison avec le BAP de référence. L'étude de la porosité accessible à l'eau des différents mélanges des BAP à base des fines issues du sable de concassage montre l'effet positif des fillers calcaires sur la diminution de la porosité. Un taux de $20 \%$ de fines dans le sable peut diminuer la porosité de $1.8 \%$.

- Un sable de concassage contenant $20 \%$ de fines permet de réduire le coefficient de migration des ions de chlorures dans les BAP.

D'une manière générale, cette étude portée sur les bétons autoplaçants à base de sable de concassage a mis en évidence que ces derniers possèdent des caractéristiques acceptables en termes de résistance et de durabilité.

\section{References}

1. B. Menadi, S. Kenai, J. Khatib , A. Ait-Mokhtar, Strength and durability of concrete incorporating crushed limestone sand, Construction and Building Materials 23 (2009):625-633.

2. B._Felekoglu. Utilisation of high volumes of limestone quarry wastes in concrete industry (selfcompacting concrete case). Resour. Conserv. Recycling 2007:1-22.

3. E. Meziane, $\mathrm{K}$ Ezziane, $\mathrm{S}$ Kenai, A Kadri. Mechanical, hydration, and durability modifications provided to mortar made with crushed sand and blended cements, Journal of Adhesion Science and Technology, 29, Issue 18(2015):1987-2005.

4. Y. Benachour, C.A, Davy., F. Skoczylas, H. Houari, Effect of a high calcite filler addition upon microstructural, mechanical, shrinkage and transport properties of a mortar. Cement and Concrete Research 38; (2008): 727-736.

5. H. Okamuara, K. Maekawa, K. Ozawa, High performance concrete. 1st ed. Tokyo: Gihoudou Pub.; 1993.

6. NF P18-459. Essai pour béton durci - Essai de porosité et de masse volumique, Mars 2010.

7. XP P18-462.Essai sur béton durci - Essai accéléré de migration des ions chlorure en régime nonstationnaire - Détermination du coefficient de diffusion apparent des ions chlorure. Juin 2012.

8. T. Celik , M. Khaled, Effects of crushed stone dust on some properties of concrete, Cement and Concrete Research, Vol. 26, No. 7, pp. 1121-1130, 1996. 